\title{
Psicoterapia basada en la Mentalización como tratamiento para Trastornos de Personalidad Borderline: Revisión teórica de los postulados de Fonagy
}

\section{Psychoterapy based on Mentalization as Borderline Personality Disorders treatment: Theorical review of Fonagy's principles}

\author{
María Josefina Escobar ${ }^{1} \quad$ María Pía Santelices \\ Gloria Peláez Elizalde \\ Pontificia Universidad Católica de Chile \\ (Rec: junio 2012 - Acep: marzo 2013)
}

\begin{abstract}
Resumen
Este artículo es una revisión teórica de los postulados de Peter Fonagy respecto a los Trastornos de Personalidad Borderline (TPB) y su propuesta de abordaje a través de la psicoterapia basada en la mentalización. A partir de la revisión se concluye que el modelo planteado para comprender el desarrollo de TPB y los lineamientos para realizar intervenciones psicoterapéuticas, resultan en gran medida un aporte al manejo de estos pacientes. Ya que el mismo ha mostrado empíricamente ser efectivo en la disminución de riesgo suicida y conductas de autoagresión; siendo estos los síntomas de mayor severidad del trastorno.

Palabras claves: mentalización, trastorno de personalidad borderline, psicoterapia, apego desorganizado
\end{abstract}

\begin{abstract}
This article is a theoretical review of the principles of Peter Fonagy about borderline personality disorder (BPD) and its proposed approach through the mentalization-based psychotherapy. From the review it is concluded that the proposed model for understanding the development of TPB and guidelines for psychotherapeutic interventions, are both a key contribution to the management of these patients. The same model and guidelines have proven empirically to be effective in reducing suicide risk and self-harm behaviors; which are the more severe symptoms of the disorder.
\end{abstract}

Key words: mentalization, borderline personality disorder, psychotherapy, disorganized attachment

\footnotetext{
Correspondencia a: Avda.Vicuña Mackenna 4860, Macul, Santiago, Chile. Escuela de Psicología. E-mail: mjescoba@gmail.com.
} 


\section{Introducción}

Estudios internacionales muestran que el trastorno de personalidad borderline (TPB) se encuentra aproximadamente en el $2 \%$ de la población global, en el $10 \%$ de los pacientes psiquiátricos ambulatorios y en el $20 \%$ de pacientes psiquiátricos ingresados (Lenzenweger, et al, 2007; Coid, et al, 2006; Lieb, et al, 2004; Comtois, et al, 2003). De acuerdo al estudio multicéntrico de la OMS sobre "Síntomas Psicológicos en Atención Primaria de Salud", se encontró que en Chile el TPB está presente en el 2,7\% de la población general (Florenzano, et al., 2002).

Un meta-análisis reciente de 13 estudios empíricos que examinan el tipo de apego que presentan los individuos con TPB, concluyó que hay una fuerte asociación entre TPB y apego inseguro; siendo los subtipos de apego más característicos: "preocupado o ambivalente" (preoccupied), "temeroso" (fearful) y "no resueltos o desorganizados" (unresolved), (Agrawal, Gunderson, Holmes \& Lyons-Ruth, 2004). Estos últimos se caracterizan por tener un déficit en la capacidad de mentalización, entendida como una dificultad en comprender los estados mentales de sí mismo y de los demás (Fonagy, 2000; Fonagy, Target, Gergely, Allen \& Bateman, 2003).

De este modo, se hace relevante el planteamiento de un modelo explicativo teórico que pueda dar cuenta de la relación entre el TPB con el apego inseguro y el déficit en la mentalización. Fonagy \& Fonagy, et al. $(2000,2003)$ sugieren que un camino para comprender el TPB es en términos de fracaso en el desarrollo de una base segura, que es crucial en el desarrollo de la capacidad de mentalización. Es así como dichos autores consideran que el apego desorganizado provocaría en el niño un déficit en la capacidad de mentalización y esto se vería manifestado posteriormente en un TPB.

Lo central al pensar en este modelo explicativo propuesto por Fonagy, es la posibilidad de que este mismo sea considerado como un aporte para el tratamiento del TPB, un trastorno que puede ser muy severo y difícil de abordar. Fonagy \& Bateman, (2003) apoyados en este modelo teórico proponen como alternativa de abordaje la "Terapia basada en la mentalización".

El objetivo de esta revisión es describir los postulados teóricos de Fonagy, para luego dar cuenta de la propuesta que realiza en torno a cómo abordar el déficit en la mentalización de pacientes con TPB. Así mismo, se mostrará evidencia empírica que respalde la efectividad de la terapia basada en la mentalización para este tipo de trastorno.

\section{Mentalización}

El constructo de mentalización fue introducido por Fonagy para definir la capacidad de entender el comportamiento propio y de los otros en términos de estados mentales (Fonagy, Steele, Steele, Higgit \& Moran, 1991).

Este constructo surge a partir de una articulación de conceptos psicoanalíticos y la teoría del apego. En la base de la mentalización está el intento de comprender la propia mente y la de los otros a partir de estados mentales, tales como intenciones, sentimientos, pensamientos, deseos y creencias; con la finalidad de dar sentido y anticipar las acciones de cada uno (Fonagy $\&$ Target, 1997). Gracias a esta capacidad uno puede entender que el comportamiento propio y el de los otros están ligados de modo significativo y predecible a sentimientos e intenciones subyacentes, que pueden ser dinámicos y cambiantes (Fonagy, Gergely, Jurist \& Target, 2002). La mentalización integraría procesos cognitivos, ya que involucra un proceso metacognitivo y afectivo, en tanto implica la capacidad de contener, regular, experienciar y dar sentido a las emociones y sentimientos (Slade, 2005).

La mentalización se desarrolla en el contexto de las relaciones del niño con los adultos significativos, especialmente en relaciones de apego seguro. Una relación segura le ofrece la posibilidad de explorar la mente del adulto, cuando este le atribuye estados mentales y lo trata como un agente mental (Fonagy et. al., 2002). La capacidad de mentalizar fue operacionalizada por Fonagy bajo el término de Función Reflexiva (Fonagy et.al., 2002).

La mentalización es considerada como una habilidad fundamental del ser humano en tanto posibilita el dar sentido a las experiencias propias y de los otros. Tener una postura mentalizadora implica tener una perspectiva psicológica que permite, por una parte, reconocer que existe un mundo interno que da forma al comportamiento, y por otra, comprender que este mundo interno es una representación que se relaciona, aunque no es idéntica, al mundo externo que modela (Wallin, 2007). Esta postura mentalizante tiene implicancias en la relación que se ejerce entre un adulto y un niño, en tanto se asume que la mentalización del adulto se pone en juego en el contexto de la relación de apego que desarrolle con el niño, y que sería un mecanismo fundamental en el desarrollo de la mentalización de este último. 


\section{Mentalización y TPB: déficit en la mentalización}

Fonagy, Target, Gergely, Allen \& Bateman (2003) han propuesto que algunos individuos con TPB son aquellas víctimas de maltrato infantil que lo afrontaron rechazando captar los pensamientos de sus figuras de apego, evitando así pensar en los deseos de sus cuidadores de hacerles daño.

Así estos individuos que sufren un trauma precoz pueden inhibir defensivamente su capacidad para mentalizar. Así pues, algunas de las características de los TPB estarían basadas en dicha inhibición (Fonagy \& Bateman, 2008; Fonagy et al., 2003; Fonagy, 2000).

La explicación para dicho fenómeno es que la proximidad mental en los niños víctimas de abusos se hace insoportablemente dolorosa, y la necesidad de cercanía se expresa en el nivel físico. De este modo, el infante puede paradójicamente sentirse impulsado a acercarse físicamente al abusador. En esta contradicción de búsqueda de proximidad tanto a nivel mental, como a nivel físico, radica el apego desorganizado que se observa en infantes maltratados (Fonagy, 2000).

Fonagy (2000) señala que de esta manera el ambiente familiar de maltrato socava la capacidad de mentalizar del infante, ya que el reconocimiento del estado mental del otro puede ser peligroso para el self en desarrollo. El infante que reconoce el odio o la violencia que implican los actos de violencia de sus progenitores, se ve forzado a verse a sí como carente de valor o como no digno de ser querido. De este modo, el significado de los estados intencionales es negado o distorsionado. Los padres abusadores a menudo exigen creencias o sentimientos opuestos a su conducta, y el infante no puede poner a prueba o modificar las representaciones de los estados mentales, por lo que se vuelven rígidas e inapropiadas. Por último, el mundo público donde la función reflexiva es común, que podría generar un modo alternativo de experimentarse a sí mismo, es mantenido rígidamente al margen del contexto de apego (Fonagy, 2000).

La función reflexiva y su contexto de apego son la base de la organización del self. La internalización de la imagen que el/la cuidador/a tiene del infante como un ser intencional es capital. En caso de maltrato la intencionalidad hostil del cuidador excluye una imagen del self coherente. La experiencia interna no encuentra la comprensión externa, permanece sin nombrar, confusa; y el afecto no contenido genera mayor desregulación. En el caso de algunos infantes maltratados, ese otro no será un otro neutral sino un torturador. Una vez internalizada esta representación "ajena" tendrá que ser expelida porque es persecutoria. El individuo, cuando está solo, se siente inseguro y vulnerable por la proximidad de una representación torturadora y destructiva de la que no puede escapar porque se experimenta desde adentro del self en lugar de afuera del self. A menos que su relación permita la externalización, se siente casi literalmente en riesgo de desaparecer, de la fusión psicológica y de la disolución de todos los límites entre sí y el otro (Fonagy, 2000).

\section{Sintomatología TPB y apego desorganizado}

Fonagy (2000) \& Fonagy, et al. (2002) presenta una serie de síntomas propios del TPB, que pueden ser explicados a partir de las experiencias tempranas de trauma que producen un estilo relacional desorganizado:

1. El sentido inestable del self es consecuencia de la ausencia de capacidad reflexiva. Un sentido del self estable sólo puede ser ilusorio cuando el self "ajeno" es externalizado en el otro y controlado allí. El individuo es, entonces, un agente activo que toma el control, al margen de la fragilidad del self. El alto precio pagado es que al forzar al otro a comportarse como si fuera parte de su propia representación interna, el potencial para una relación "real" se pierde. De este modo, el paciente está preparando el camino para el abandono.

2. La impulsividad de estos pacientes puede ser debida de igual forma a: a) falta de conciencia de sus estados emocionales asociada con la ausencia de una representación simbólica de los mismos, y b) la dominancia de estrategias físicas pre-mentalísticas centradas en la acción, particularmente en las relaciones amenazantes.

3. La inestabilidad emocional y la irritabilidad requieren que pensemos sobre la representación de la realidad en los pacientes con TPB. La ausencia de mentalización reduce la complejidad de esta representación; sólo es posible una versión de la realidad, no pueden existir falsas creencias (Fonagy \& Target, 1996).

4. El riesgo de suicidio. Esto alerta a los clínicos sobre los modelos de apego desorganizado con los que tales pacientes son forzados a vivir. Cuando se necesita al otro para la propia coherencia, el abandono significa la reinternalización de la intolerable autoimagen "ajena", y la consecuente destrucción del self. 
5. La escisión, la representación parcial del otro (o del self) es un obstáculo común para la comunicación con este tipo de pacientes. Dado el imperativo de alcanzar representaciones coherentes, la solución es escindir la representación del otro (Gergely, 1997), primariamente una identidad idealizada y una persecutoria. Encuentra imposible emplear ambas representaciones simultáneamente. Tal escisión hace posible al individuo crear imágenes mentalizadas de otros pero que son inexactas, ultra simplificadas y sólo permiten una ilusión de un intercambio interpersonal mentalizado.

6. El sentimiento de vacío que acompaña gran parte de sus vidas. Como una consecuencia directa de la ausencia de representaciones secundarias de los estados del self y de la superficialidad con la que se experimentan a los demás y sus relaciones. El abandono de la mentalización crea un profundo sentimiento de aislamiento. Para experimentar el estar con otra persona, tiene que haber una mente; para sentir la continuidad entre el pasado y el presente, esta conexión es proporcionada por los estados mentales.

\section{Terapia basada en la mentalización}

El núcleo de la terapia psicológica con pacientes con trastorno de personalidad severo es la facilitación de los procesos reflexivos (Fonagy \& Bateman, 2008; Fonagy, 2000).

La meta del tratamiento para TPB es la mentalización en orden a fomentar el desarrollo de representaciones internas estables. De esta manera, fomentar la formación de un sentido coherente del self y posibilitar que el paciente pueda establecer relaciones más seguras en que las motivaciones de su self y la de los otros sean mejor comprendidas (Fonagy \& Bateman, 2003).

Fonagy \& Bateman (2008) proponen que los objetivos que se buscan alcanzar con el paciente son que desarrolle un mejor conocimiento sobre cómo piensa y siente sobre sí mismo y los otros; la forma en que dirige sus respuestas a los demás; y cómo "errores" en la comprensión de sí mismos y de los otros, conducen a acciones en un intento de mantener la estabilidad y a dar sentido a sentimientos incomprensibles. El terapeuta, por su parte, tiene que asegurarse de que el paciente sea consciente de estos objetivos y tenga conocimiento del proceso de la terapia en sí misma, es decir, que el paciente comprenda el núcleo del tratamiento. Todo esto, sobre el supuesto de que el proceso de mentalización sólo puede ser desarrollado, si la estructura del tratamiento es cuidadosamente definida.

Para alcanzar los objetivos hay algunas tácticas en esta terapia. Primero, como ya se anunció, la importancia de la claridad en los objetivos y expectativas terapéuticas, ya que los pacientes con TPB juzgan la motivación de los otros en los resultados. Segundo, la comprensión, la interpretación y otras intervenciones deben estar basadas en considerar la estabilidad de la estructura del self del paciente. Tercero, la proximidad mental es un prerrequisito para una terapia eficaz y es conseguida por intervenciones contingentes y marcadas. Cuarto, aceptar la necesidad reconducirle algunos aspectos relativos al "self ajeno", como es en la proyección, en la contratransferencia, y en la escición. Por último, es necesario el reconocimiento de la ausencia de representaciones simbólicas en estos pacientes (Fonagy \& Bateman, 2003).

Este tipo de terapia se realizaría fundamentalmente en tres fases, cada una de ellas con objetivos claramente delimitados (Fonagy \& Bateman, 2008). Dentro de los objetivos generales de la fase inicial, se podría mencionar: la evaluación de la capacidad de mentalización del paciente y el comprometerlo con el tratamiento. Como procedimientos específicos en la etapa inicial del tratamiento se incluyen: la psicoeducación, dar un diagnóstico, establecer una jerarquía de objetivos terapéuticos, estabilizar los problemas sociales y de comportamiento, revisar los medicamentos, y definir una vía de crisis. En una segunda fase, el objetivo del trabajo terapéutico es estimular cada vez más la capacidad de mentalización. Y en la etapa final, se prepara al paciente para poner fin al tratamiento; esto requiere que el terapeuta se centre en los sentimientos de pérdida con que se termina el tratamiento y la manera de mantener las ganancias que han realizado, así como en el desarrollo de un adecuado programa de seguimiento adaptado a las necesidades particulares del paciente.

A lo largo del programa del tratamiento es necesario (1) clarificar y nombrar los sentimientos continuamente; (2) entender de inmediato los estados emocionales precipitantes, con las circunstancias presentes; (3) entender los sentimientos en el contexto previo y presente de las relaciones; (4) expresar las emociones de manera apropiada y en el contexto de las relaciones; y (5) entender las respuestas a las reacciones en el contexto de las relaciones (Fonagy \& Bateman, 2003). Para conseguir esto, el paciente necesita la ayuda del terapeuta en el ejercicio de comprender las emociones y acciones propias y de los demás, a partir de las circunstancias en las que se producen. 
La efectividad de esta propuesta ha sido presentada mediante estudios de caso único, tratados con esta modalidad de psicoterapia (Fonagy, Bateman, Ryle, \& Kerr, 2007) y además, a través de los resultados de un estudio aleatorio (Fonagy \& Bateman, 2009). En este estudio la muestra fue de 134 pacientes con TPB; de los cuales, 71 pacientes fueron tratados con psicoterapia basada en la mentalización y 63 pacientes contaron con un tratamiento clínico estructurado. Los resultados obtenidos fueron favorables en ambas condiciones, presentando mejores resultados en las terapias basadas en la mentalización en relación al riesgo suicida y en la disminución de las conductas de autoagresión. Debido a la diferencia en el foco de ambos tratamientos, la psicoterapia basada en la mentalización mostró cambios más rápidos en el estado de ánimo, las medidas interpersonales y el ajuste social, en comparación con el grupo que recibió el tratamiento clínico estructurado.

\section{Discusión}

Los postulados teóricos planteados por Fonagy y colaboradores para comprender el desarrollo de TPB y los lineamientos o directrices para realizar intervenciones psicoterapéuticas, resultan en gran medida un aporte clínico al manejo de estos pacientes. La modalidad de psicoterapia basada en la mentalización propuesta para el abordaje de pacientes con TPB, busca reparar la falla y las carencias en la función mentalizante, es decir, en el déficit de la mentalización que tiene origen en los primeros vínculos afectivos, lo cual es un proceso complejo de revertir. Y no solo disminuir los síntomas foco de los tratamientos clínicos estructurados.

Sin embargo, esta modalidad de psicoterapia no pretende explicar toda la complejidad del trastorno, por lo que no descarta la parte biológica comprometida en el mismo y la inclusión de psicofármacos; dependiendo de la gravedad del caso. Es por esto que la fase inicial se plantea como una etapa de gran importancia ya que toma en consideración la revisión de diversos ámbitos. Para así poder continuar con el foco principal que es el fomento de la capacidad reflexiva.

Asimismo, es en esta primera fase que el terapeuta debe generar un fuerte vínculo con el paciente. Ya que, como se ha expuesto, estos pacientes sufren de una carencia en las experiencias vinculares tempranas, donde hubo un cuidador no adecuado que no le permitió desarrollar su capacidad de mentalizar. A través de este nuevo vínculo con el terapeuta podrá reparar el déficit en la mentalización. Por ello, es fundamental lograr establecer una alianza basada en la seguridad del apego. Una vez lograda la alianza terapéutica, el terapeuta guiará y acompañará al paciente en un proceso de elaboración y simbolización de su historia personal, de tal manera que el paciente logre comprender su historia personal en términos de estados mentales de sí mismo y de los otros, para así establecer nexos entre sus experiencias de infancia y sus experiencias actuales.

Respecto a los hallazgos empíricos de esta psicoterapia, se destaca el hecho de que ha mostrado ser efectiva en la disminución de los síntomas más severos de estos pacientes, evidenciando la disminución del riesgo suicida y las conductas autoagresivas (Fonagy \& Bateman, 2009; Fonagy, Bateman, Ryle \& Kerr, 2007). Así como, un mayor ajuste social y adecuación en las relaciones interpersonales; siendo estos últimos foco de conflicto y motivo de consulta frecuente en estos de pacientes.

En resumen, la propuesta de Fonagy y colaboradores brinda herramientas concretas para un abordaje efectivo de los pacientes con TPB. Existen estudios que respaldan la eficacia de la Terapia basada en la mentalización, lo cual constituye un gran aporte para el quehacer del psicoterapeuta. Además, se propone, como objetivo central del tratamiento, abordar y trabajar el déficit en la capacidad de mentalización en pacientes con TPB. Se busca otorgar un espacio para reparar carencias tempranas, mejorar su calidad de vida, así como la de su entorno. Finalmente, el tratamiento destaca la importancia de brindar herramientas para que pueda mantener los cambios una vez concluido el proceso psicoterapéutico.

\section{Referencias}

Agrawal, H., Gunderson, J., Holmes, B. \& Lyons-Ruth, K. (2004). Attachment Studies with Borderline Patients: A Review. Harvard Rev Psychiatry. 12, (2), 94-104.

Coid, J., Yang, M., Tyrer, P., Roberts, A. \& Ullrich, S. (2006). Prevalence and correlates of personality disorder in Great Britain. $\mathrm{Br} J$ Psychiatry, 188, 423-431

Comtois, KA., Russo, J., Snowden, M., Srebnik, D., Ries, R. \& Roy-Byrne, P. (2003). Factors associated with high use of public menthal health services by persons with borderline personality disorder. Psychiatr Serv, 54, 1149-1154.

Florenzano, R., Weil, K., Cruz, C., Acuña, J., Fullerton, C., Muñiz, C., Leighton, C. \& Marambio, M. (2002). Personalidad limítrofe, somatización, trauma y violencia infantil: un estudio empírico. Revista Chilena de Neuro-psiquiatría, 40, 335-340.

Fonagy, P. (2000). Apegos patológicos y acción terapéutica. Trabajo presentado en el "Grupo psicoanalítico de discusión sobre el desarrollo", en la reunión de la Asociación Psicoanalítica Americana, Washington DC, 13 de mayo de 1999. Revista Aperturas Psicoanalíticas $N^{\circ} 004$ 
Fonagy, P. \& Bateman, A. (2003). The development of an attachmentbased treatment program for borderline personality disorder. Bulletin of the Menninger Clinic. 67, 3, 187-211.

Fonagy, P. \& Bateman, A. (2006) Mechanisms of change in mentalization-based treatment of BPO. Journal of Clinical Psychology. 62, (4), 411-430

Fonagy, P. \& Bateman, A. (2007). Mentalizing and borderline personality disorder. Journal of Mental Health, Special issue: Developing new treatments of personality disorder. 16, (1), 83-101.

Fonagy, P. \& Bateman, A. (2008). Mentalization-based treatment for BPD. Social Work in Mental Health. 6, (1 /2), 187-201.

Fonagy, P. \& Bateman, A. (2009). Randommized controlled trial of outpatient mentalization-based treatment versus structured clinical management for borderline personality disorder. Am J Psychiaty. 166(12), 1355-1364.

Fonagy, P., Bateman, A., Ryle, A. \& Kerr, I. (2007). Psychotherapy for borderline personality disorder: Mentalization Based Therapy and Cognitive Analytic Therapy compared. International Review of Psychiatry, 19(1), 51-62.

Fonagy, P., Steele, H.,. Steele, M., Higgit, A \& Moran, G. (1991). The capacity for understanding mental states: The reflective self in parent and child and its significance for security of attachment. Infant Mental Health, 13, 200-217.
Fonagy, P. \& Target, M. (1997). Attachment and reflective function: Their role in self-organization. Development of Psychology, 9, 679-700.

Fonagy, P., Target, M., Gergely, G., Allen, J. \& Bateman, A. (2003). The developmental roots of borderline personality disorder in early attachment relationships: A theory and some evidence. Psychoanalytic Inquiry, Special issue: Infant research. 23(3), 412-459.

Fonagy, P., Target, M., Gergely, G. \& Jurist, E. (2002). Affect Regulation, mentalization and the development of the self. New York: Other Press.

Lansky, M. (2003). Discussion of Peter Fonagy et al.'s 'The developmental root of borderline personality disorder in early attachment relationships: A theory and some evidence Psychoanalytic Inquiry, Special issue: Infant research. 23(3), 460-472.

Lenzenweger, MF., Lane, MC., Loranger, AW. \& Kessler, RC. (2007). DSM-IV Personality disorders in the National Comorbidity Survey Replication. Biol. Psychiatry, 62, 553-564.

Lieb, K., Zanarini, MC. \& Schamahl, C. (2004). Borderline Personality disorder. Lancet, 364, 453-461.

Slade, A. (2005). Parental reflective functioning: An introduction. Attachment and Human Development, 7, 3, 269-281.

Wallin, D. J. (2007) Attachment in Psychotherapy. New York: The Guilford Press. 